\title{
Are visually presented one-syllable words integral stimuli?
}

\author{
WAYNE P. SILVERMAN \\ New York State Institute for Basic Research in Mental Retardation, Staten Island, New York 10314
}

\begin{abstract}
Subjects discriminated between sequences of four letters on the basis of the initial letter ( $\mathrm{a}$ "P" or an "R"). Sequences formed one-syllable words or unpronounceable nonwords. All subjects performed four different discriminations (word simple, word filtering, nonword simple, and nonword filtering). Simple discriminations were based on the first letter of a sequence, with all other letters held constant. Filtering tasks required the same discrimination, but also varied the third letter (variation in only the first letter was relevant to response selection). Results showed no differences between any condition in either reaction time or error rate, suggesting that both monosyllabic words and unpronounceable letter sequences were processed by their component letters. Results are consistent with suggestions that unitization differences between visually presented pronounceable and unpronounceable letter sequences may be due to phonemic recoding.
\end{abstract}

Spoehr and Smith (1973), investigating tachistoscopic recognition of letter sequences, showed that responses are more accurate when letters form words than when they form random sequences. This is a common finding that will be referred to herein as the "word superiority effect" (WSE). [The reader is referred to Krueger's (1975) recent review of this area.] Spoehr and Smith suggested that the WSE is found because pronounceable letter sequences are processed in multiletter perceptual units, while unpronounceable sequences are analyzed letter by letter. Thus, the WSE is caused by a differentiation between words and nonwords in an early "unitization" process, with measured performance differences due to the size of the respective perceptual units.

Silverman (1976) tested the Spoehr and Smith (1973) theory by attempting empirical measurement of the perceptual unit size for words and nonwords. The method employed was based on Garner's (1974) definitions of integral and separable stimuli. (Garner suggested that some stimuli are processed as single units, and called these integral stimuli. In contrast, separable stimuli are analyzed by their component units, or features. As a first approximation, these concepts served to define tasks sensitive to unitization differences.)

Garner pointed out several empirical properties that will differentiate between integal and separable stimuli. One can be observed in two-choice discrimination tasks (e.g., Biederman \& Checkosky, 1970). When integral stimuli are processed and the subjects' task is to discriminate between two possible alternatives differing on either one or two relevant dimensions, performance will be better for pairings differing on two dimensions than for those differing on a single dimension. This performance improvement, termed a redundancy gain, will

I thank A. L. Hill for helpful comments on an earlier draft of this paper. Address: 1050 Forest Hill Road, Staten Island, New York 10314. not occur when separable stimuli are processed; Garner's framework predicts that, given separable stimuli, discriminations between pairs differing on two dimensions should be no better than the easier, yet no worse than the more difficult unidimensional discrimination.

To see if one-syllable words are processed as single units, Silverman (1976) modified the Biederman and Checkosky (1970) speeded discrimination method for use with letter sequence stimuli. A letter was arbitrarily defined as a feature, and subjects were required to discriminate between two sequences differing in either one or two letters. Results showed: (1) no difference between words and nonwords when discriminations were based on a single letter [replicating earlier results (e.g., Massaro, 1973), where subjects knew the locus of critical information before test trials]; (2) no redundancy gain for nonword discriminations differing in two letters; and (3) a redundancy gain for word discriminations differing on two letters. This pattern of results implies that nonwords were processed letter by letter, while one-syllable words were processed as units, and is consistent with the Spoehr and Smith (1973) theory.

An additional contrast also suggested that onesyllable words may be integral stimuli (Silverman, 1976). Gottwald and Garner (1975) showed that a performance advantage favoring integral over separable stimuli occurs in a condensation task (Fitts \& Biederman, 1965), where no single stimulus dimension furnishes sufficient task-relevant information for correct performance. This advantage occurs although individual feature discriminability is matched for the two stimulus types. Silverman found this predicted performance difference between words and nonwords.

While the data summarized above imply that onesyllable words are integral stimuli and nonwords are separable stimuli, there are problems with this hypothesis. A great deal of evidence suggests that stimuli consisting of features separated spatially (as are the components of printed letter sequences) are separable 
stimuli (e.g., Lockhead, 1966). Therefore, before the words can be identified as integral stimuli, further evidence of systematic word-nonword performance differences should be deomonstrated within the Garner (1974) framework.

The integral-separable distinction is based on an empirical system of converging operations. The differential redundancy gains described above and the performance difference in the condensation task are two of these operations. A third measurable difference between integral and separable stimuli occurs in a filtering task (Fitts \& Biederman, 1965), where subjects must disregard variation in an irrelevant dimension in order to maximize performance. Subjects have no difficulty doing this when presented with separable stimuli, but cannot effectively filter irrelevant information when discriminating between integral stimuli. The present experiment was designed to detect differential interference between words and nonwords in a filtering task. If one-syllable words are integral stimuli and nonwords are separable stimuli, results should show: (1) no wordnonword differences when discriminations are based on a single letter; (2) no difference between filtering and simple discriminations for nonwords; and (3) poorer performance in the filtering task for words. In contrast, if the words are separable stimuli, there should be no filtering task interference for either stimulus category.

\section{METHOD}

The stimuli, apparatus, and procedure are described in detail elsewhere (Silverman, 1976). Below is a summary of the method.

The stimuli were strings of four letters. For the word condition the stimuli were PAPE, PARE, RAPE, and RARE. The nonwords were formed by substituting consonants for the vowels of the words. Consonants were chosen to maximize the visual similarity between the word and nonword stimuli (as judged by three independent raters). The nonwords were PXPF, PXRF, RXPF, and RXRF. All stimuli were presented in a two-channel tachistoscope and subtended horizontal visual angles of $1 \mathrm{deg} 4 \mathrm{~min}$ when viewed by subjects.

Eight adults participated as subjects. All had previous experience as subjects in reaction time (RT) tasks similar in procedure to the present experiment. Each subject viewed four blocks of test trials, preceded by a block of 10 practice trials. Each condition consisted of a block of 36 trials, randomly ordered, the first 12 counting as additional practice. Subjects discriminated between PAPE and RAPE in the practice block. For the word-simple discrimination, subjects discriminated between PARE and RARE. For the word-filtering discrimination, subjects responded with one hand if shown PARE or PAPE and with the other if shown RARE or RAPE. Required performance for nonwords was analogous to the word condition.

Subjects viewed all four test blocks. Block order was counterbalanced in a Latin square design. An experimental session lasted approximately $25 \mathrm{~min}$. (Only "first-letter" simple and filtering conditions were used in this design, as evidence for or against interference in this task is sufficient for making the integralseparable distinction.)

Each trial consisted of: (1) a verbal ready signal; (2) the onset of a 1.000-sec warning stimulus (a rectangle surrounding the area where the stimulus was to appear), which also functioned as a fixation field; (3) the onset of the stimulus (either an " $X$ " or " $O$ " for practice trials and one of the letter sequences for experimental trials) for $30 \mathrm{msec}$; (4) the offset of the stimulus followed by a dark field; (5) the depression of one of the two response keys, stopping an electronic counter and recording the subject's RT (to the nearest millisecond) and response; and (6) the positioning of the next stimulus. No feedback was given during the intertrial interval, which was approximately $3 \mathrm{sec}$. This procedure minimized subjects' temporal; positional, and featural uncertainty, eliminated stimulus scanning eye movements, and presented subjects with an easily identified, nondegraded stimulus.

\section{RESULTS AND DISCUSSION}

The results are given in Table 1. Each RT represents the mean of the individual subjects' median RT for that condition. Analyses of variance for the RT and error rate data showed no significant main effects or interactions.

The total absence of interference in the word-filtering task suggests that visually presented one-syllable words are not integral stimuli. ${ }^{1}$ The slight trend in the data was opposite to theoretical predictions based on an integral-separable distinction between the word-nonword stimulus categories. Results indicate that both pronounceable and unpronounceable sequences must be separable stimuli as defined in Garner's framework.

In light of the present data, the earlier results obtained by Silverman (1976) raise an interesting question. Are responses selected on the basis of visual or phonemic analysis? The present failure to find any evidence of interference for words in the filtering task suggests that some processing option allowed for the redundancy gains found for words in the earlier experiments (Silverman, 1976). (Garner allows no processing options with integral stimuli.) Based on visual mechanisms, there is no obvious processing option that results in redundancy gains only for words. However, a likely option involves a visual to phonemic recoding, with subjects processing "units" that maximize subjective efficiency. Such a scheme predicts that one-syllable words can be processed as single units, and is described in detail by Spoehr and Smith (1975). This description of information flow is also compatible with the Garner (1974) theory.

The question raised here involves the predictability of task nature (i.e., predominantly visual or phonemic). Estes (1975) proposed that response selection should be based on visual processing when observers know what critical visual features to scan for before stimulus presentation, and efficient performance can result from visual analysis. The Silverman (1976) procedure not only

Table 1

Mean RTs (Milliseconds) and Error Rates for the Various Experimental Conditions

\begin{tabular}{cccccc}
\hline & \multicolumn{2}{c}{ RT } & & \multicolumn{2}{c}{$\%$ Error } \\
\cline { 2 - 3 } \cline { 5 - 6 } Discrimination & Words & Nonwords & & Words & Nonwords \\
\hline Simple & 497 & 495 & & 3.1 & 5.7 \\
Filtering & 492 & 502 & & 5.7 & 8.8 \\
\hline
\end{tabular}


gave subjects advance knowledge of the critical features, but also minimized positional uncertainty by holding critical item location constant for an entire test block. Therefore, the Estes model predicts that processing in the experiments showing redundancy gains for words should be visual. Such a prediction is both feasible and intuitively logical but, given the data, tends to conflict with Garner's framework. It therefore seems necessary to either modify the conditions where redundancy gains can occur given separable stimuli, or concede that accurately predicting the level of analysis preceding response selection when subjects process visually presented letter sequences is a much more difficult task than Estes (1975) suggests.

\section{REFERENCES}

Biederman, I., \& Checkosky, S. Processing redundant information. Journal of Experimental Psychology, 1970, 83, 486-490.

Estes, W. K. Memory, perception, and decision in letter identification. In R. Solso (Ed.), Information processing and cognition: The Loyola symposium. Potomac, Md: Lawrence Erlbaum, 1975.

Fitts, P., \& Biederman, I. SR compatibility and information reduction. Journal of Experimental Psychology, 1965, 69, 408-412.

GARNER, W. R. The processing of information and structure. Potomac, Md: Lawrence Erlbaum, 1974.

GARNER, W. R., \& FELFOLDY, G. Integrality of stimulus dimensions in various types of information processing. Cognitive Psychology, 1970, 1, 225-241.

GotTwALD, R., \& GARNER, W. R. Filtering and condensation tasks with integral and separable dimensions. Perception \& Psychophysics, 1975, 18, 26-28.

KRUEGER, L. Familiarity effects in visual information processing. Psychological Bulletin, 1975, 82, 949-974.

LOCKHEAD, G. Effects of dimensional redundancy on visual discrimination. Journal of Experimental Psychology, 1966, 72, 95-104.

Massaro, D. Perception of letters, words, and nonwords. Journal of Experimental Psychology, 1973, 100, 349-353.

Silverman, W. Can "words" be processed as integrated units? Perception \& Psychophysics, 1976, 20, 143-152.

SpoenR, K. T., \& Smith, E. E. The role of syllables in perceptual learning. Cognitive Psychology, 1973, 5, 71-89.

Spoehr, K. T., \& SMith, E. E. The role of orthographic and phonotactic rules in perceiving letter patterns. Journal of Experimental Psychology: Human Perception and Performance, 1975, 1, 21-34.

\section{NOTE}

1. Acceptance of a null hypothesis is a risky business; however, the Garner (1974) framework is quite explicit in requiring the presence of filtering task interference for stimuli to be classified as integral. Nevertheless, the experiment was replicated using different letter sequences and a speeded card sorting task (e.g., Garner \& Felfoldy, 1970), with results showing the same pattern as those reported herein.

(Received for publication September 3, 1976.) 